\title{
EFFECT OF CREATINE ON THE PAIN OF MUSCLE ISCHEMIA
}

\author{
By F. B. CULP AND F. W. KINARD \\ (From the Departments of Chemistry, and Physiology, Medical College of the State of South \\ Carolina, Charleston)
}

(Received for publication September 8, 1946)

The stimulus of the pain which arises from exercising ischemic muscle has been ascribed to a "factor P" (1). It has been suggested that this factor may be lactic acid, a compound of phosphorus, or creatine, or ammonia (2); phosphoric acid (3); potassium (4); lactate ion (5); or histamine (6). On the other hand, conclusions have been reached that potassium, sarcolactate, and ammonium ions are probably not responsible (6).

No investigations of the relation of creatine to the pain of muscle ischemia have been reported although such a relationship has been suggested (2). In the present study, the pain in exercising ischemic muscle was studied before and during creatine administration in order to observe the effect of creatine upon the pain process.

\section{METHODS}

Healthy male medical students were used as subjects with 6 in the control group and 8 in the experimental group. Each subject ate the usual diet of his choice and collected his total 24-hour output of urine. At 3:00 P.M. each day the subject brought the urine to the laboratory and submitted to a standard procedure (7) for the determination of the duration of exercise before the appearance of "definite" pain in the exercising ischemic muscle. The endpoint chosen was the first appearance of a definite sensation of pain in the forearm or hand after exercise had been executed at the rate of one contraction per second. The time, in seconds, was recorded by a stop watch concealed from the subject. The right arm alone was used in all experiments. Instead of simple flexion of the fingers to form a fist, the subject squeezed a gripping device against a spring tension of $1.1 \mathrm{kgm}$.

The urinary creatine and creatinine were determined in duplicate by the method of Peters (8), using the Evelyn photoelectric colorimeter for the color comparisons. Urine studies were made during both the preliminary control and the experimental periods.

Following a period of several days during which the subject became familiar with the procedure, single observations were made daily for a control period of 2 days before creatine administration was begun.

Creatine, C.P., was dissolved in water, chilled, flavored with citric acid and saccharin, and administered in daily doses of 10 grams for a period of 4 days. Creatine was given only after 2 days of preliminary studies. The control subjects were given flavored water, from a flask labelled "creatine," and were led to think that they were being given creatine. No one was informed of the grouping of subjects or of the exact nature of the experiments.

\section{RESULTS}

The exercise duration, in seconds, until the appearance. of a definite sensation of pain in the exercising ischemic muscles was recorded during the 2-day preliminary period. For each subject the mean of these two values is plotted in Figures 1 and 2 on the zero line of the control period. Each of the two values is then plotted in terms of per cent variation from the mean value. During the experimental periods, the time of appearance of pain is plotted as per cent variation from the mean value of the preliminary control period.

Control subjects. In each of the 6 subjects, the time of appearance of definite pain in the preliminary control period did not vary more than 5.5 per cent from the mean of the two values (Figure 1).

On the first day after receiving the flavored water, to be designated hereafter as "placebo," only 2 subjects (C. H.; R. M.) observed definite pain within the time range of the preliminary control period. One of these, R. M., again observed pain within the control range on the second day. The only definite trend toward uniformity of response was the relatively delayed pain appearance in five of the subjects on the fourth day of the experimental period, as shown in Figure 1.

Creatinuria was not observed in any of the control subjects at any time.

Subjects receiving creatine. On the first day of the experimental period, 5 of the 8 subjects observed definite pain either within the limits of the preliminary control period or earlier. By the fourth day, when creatine retention was at a maximum, 6 subjects observed pain later than it 


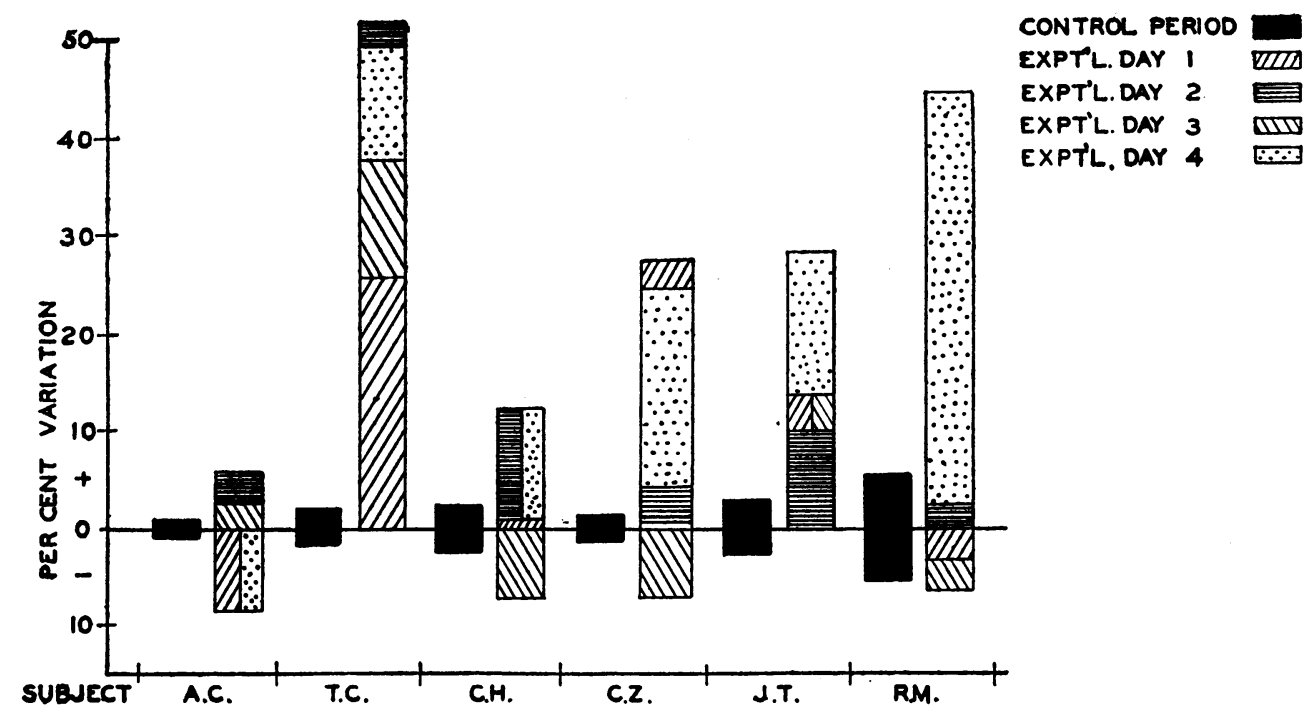

Fig. 1. Per Cent Variation in Time of Appearance of Definite Pain in Exercising Ischemic Muscle During Preliminary Control Period and During Experimental Period when Placebos were Given
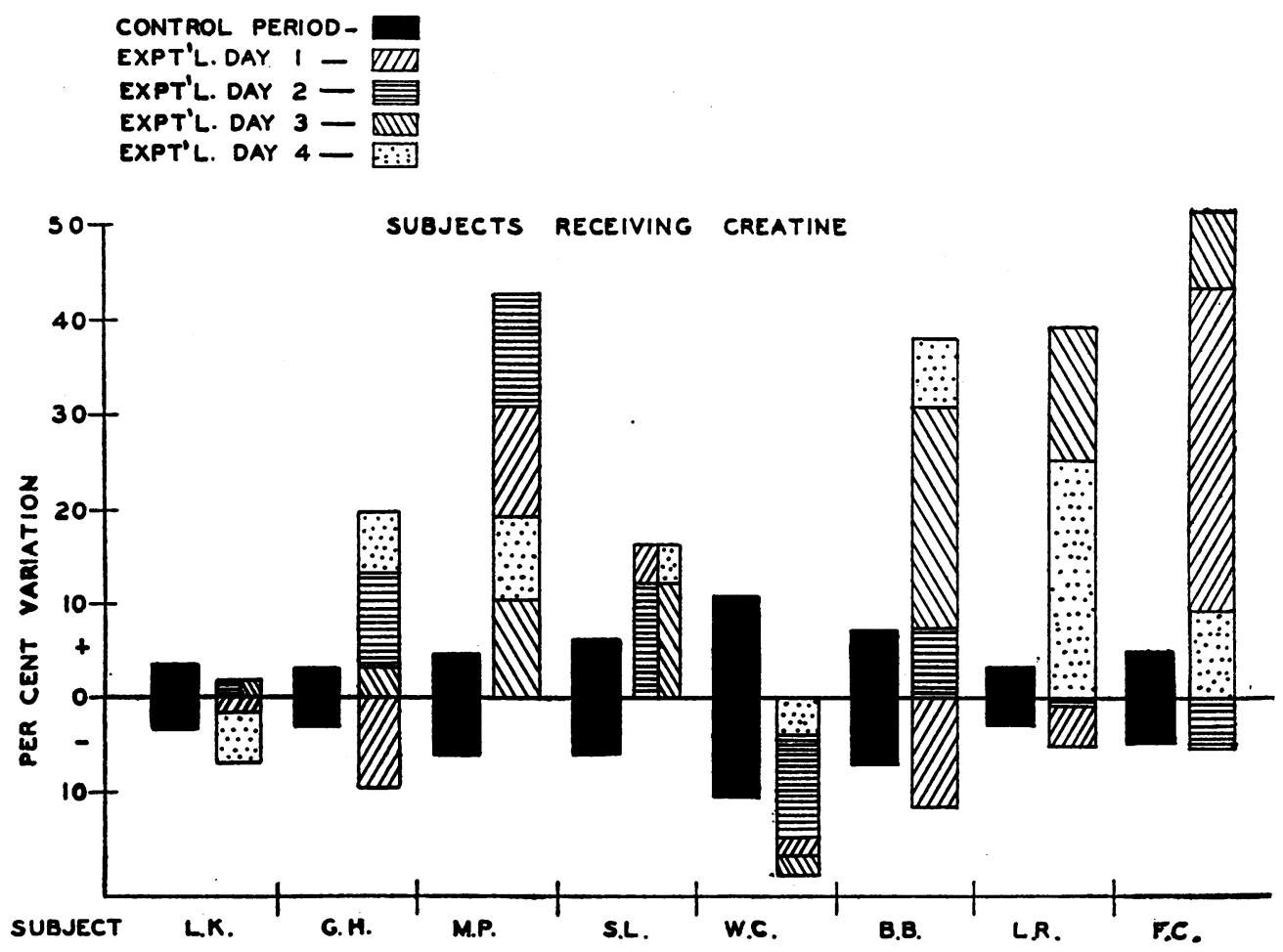

Fig. 2. Per Cent Variation in Time of Appearance of Definite Pain in Exercising Ischemic Muscle During Preliminary Control Period and During Experimental Period when Creatine was Given 


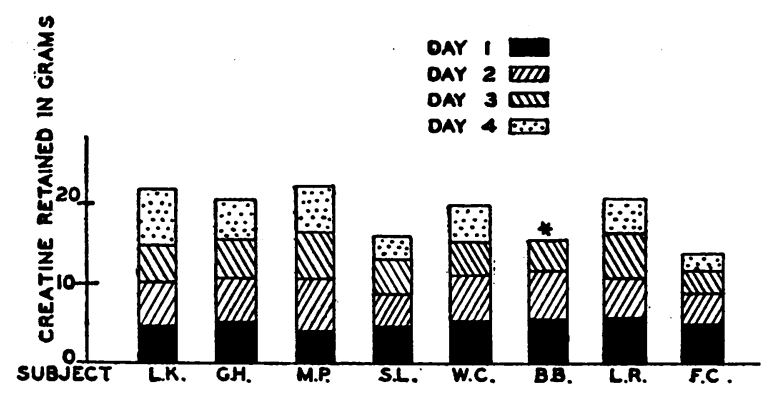

Fig. 3. Creatine Retention, in Grams, During Experimental Period when Creatine Was AdminisTERED

* Urine specimen lost.

had appeared during the control period, as shown in Figure 2.

Creatine retention. The subjects were on their usual diets, which were not creatine-free; so an arbitrary method of calculation was adopted. The creatine retained was considered to be the difference between that which had been fed and that excreted through each successive day of the experimental period. This value is recorded as the retained creatine and is plotted in Figure 3. The maximum retention was 22.60 grams of creatine in subject M. P., and the lowest was 13.79 grams in subject F. C.

\section{DISCUSSION}

It has been reported $(9,10)$ that the creatine concentration of muscle is increased by the feeding of creatine. In our subjects, there was a definite trend toward delayed appearance of pain in exercising ischemic muscle as the quantity of retained creatine increased. Perhaps, then the pain-producing phenomenon in ischemia is related to the creatine content of the muscle. However, a similar delay in pain appearance occurred in the control subjects. Since the two groups reacted in a similar manner and since the controls were not receiving creatine, it appears definite that increased creatine storage is not the fundamental cause of the delayed pain response. Training, or possibly some psychological factor, may be involved but this is mere speculation.

In comparing the amount of exercise required to produce pain in the two arms of 7 subjects, Katz, et al (11) found that pain appeared earlier in the left arm. The difference in the two arms was ascribed to training, as the right hand had been used much more frequently in the clenching exercise. We have used the right arm alone in all experiments and have made a single determination daily for not longer than 8 days. It would seem that training, under these conditions, would play not more than a minor role. In addition, several subjects showed marked shortening of the period of exercise on the first, second, or third days of the experimental period. If training is the major factor in producing the general tendency toward the longer toleration of exercise, it certainly does not influence the results in a graded uniform fashion.

\section{SUM MARY}

There was a definite trend toward delayed appearance of pain, in a majority of 8 subjects, as the quantity of retained creatine increased. However, the same tendency was observed in the control group which was receiving a placebo only. It is concluded that increased concentration of muscle creatine is not the primary cause of the delayed appearance of pain. Training may be involved in the delay but some unexplained factor, or factors, seems more likely.

\section{BIBLIOGRAPن்Y}

1. Lewis, T., Pickering, G. W., and Rothschild, P., Observations upon muscular pain in intermittent claudication. Heart, 1931, 15, 359.

2. Kissin, M., The production of pain in exercising skeletal muscle during induced anoxemia. J. Clin. Invest., 1934, 13, 37.

3. Katz, L. N., Mechanism of pain production in angina pectoris. Am. Heart J., 1935, 10, 322.

4. Harpuder, K., and Stein, I. D., Studies on the nature of pain arising from an ischemic limb. II. Biochemical studies. Am. Heart J., 1943, 25, 438.

5. Elliot, A. H., and Evans, R. D., Ischemic pain in exercising muscles. Am. Heart J., 1936, 12, 674.

6. Maison, G. L., Studies on the genesis of ischemic pain: The influence of the potassium, lactate and ammonium ions. Am. J. Physiol., 1939, 127, 315.

7. Harrison, I. B., and Bigelow, N. H., Quantitative studies of visceral pain produced by the contraction of ischemic muscle. A. Research Nerv. \& Ment. Dis., 1943, 23, 154. 
8. Peters, J. H., The determination of creatinine and creatine in blood and urine with the photoelectric colorimeter. J. Biol. Chem., 1942, 146, 179.

9. Chanutin, A., and Silvette, H., The influence of fasting and creatine feeding upon the creatine content of the tissues and blood of the white rat. J. Biol. Chem., 1928, 80, 589.

10. Chanutin, A., and Beard, H. H., A study on the effect of feeding creatine on growth and its distribution in the liver and muscle of normal mice. J. Biol. Chem., 1928, 78, 167.

11. Katz, L. N., Lindner, E., and Landt, H., On the nature of the substance ( $s$ ) producing pain in contracting skeletal muscle: Its bearing on the problems of angina pectoris and intermittent claudication. J. Clin. Invest., 1935, 14, 807. 